\title{
Characterization of silane coated hollow sphere alumina-reinforced ultra high molecular weight polyethylene composite as a possible bone substitute material
}

\author{
S ROY and S PAL* \\ School of Bioscience and Engineering, Jadavpur University, Kolkata 700 032, India
}

MS received 17 September 2001; revised 23 October 2002

\begin{abstract}
Silane coated hollow sphere alumina ceramic particles were moulded with ultra high molecular weight polyethylene (UHMWPE) to form a series of composites with alumina weight percent in the range from 15 to 50. The composites were prepared in a cylindrical mould using powder-processing technique. The composites were characterized for mechanical properties using destructive and non-destructive ultrasonic testing methods. The physical properties of the composite were determined and compared with those of cortical bone.
\end{abstract}

Keywords. UHMWPE; powder processing; non-destructive; ultrasonic; cortical bone.

\section{Introduction}

Various engineering materials have been used in orthopaedic applications for bone replacement or repair in a variety of surgical procedures. The implanted material is expected to withstand any anticipated physiological loading without dimensional changes, catastrophic brittle fracture or fracture in the longer term due to creep, fatigue or stress corrosion (Bonfield 1988). Historically, fracture fixation devices, i.e. plates and screws, which hold broken bones together during fracture healing, are usually made of metals. They maintain alignment and the stability of the implant with respect to the fractured surfaces. Despite metals having the required mechanical properties, their high modulus of elasticity results in major loads being carried through the metal plate rather than the bone. This results in the bone being inadequately loaded and consequently it resorbs. This phenomenon is described as stress shielding. Unfortunately as healing progresses or completed, rigid fixation may cause bone to undergo stress protection atrophy and osteoporosis (Woo et al 1976).

The elastic modulus of cortical bone ranges from $3 \mathrm{GPa}$ to $30 \mathrm{GPa}$ (Ravglioli and Krajewski 1992) and for the cancellous bone $0.09 \mathrm{GPa}$ to $0.23 \mathrm{GPa}$ (Park 1984), while commonly used alloys have modulus ranging from $110 \mathrm{GPa}$ ( $\mathrm{Ti}$ alloys) to $210 \mathrm{GPa}$ (316L stainless steel). The elastic mismatch of this order becomes a problem in clinical practice, which depends on the magnitude of the applied load, the nature of the interfacial bond, and

\footnotetext{
*Author for correspondence
}

the elastic constants of the interfacial materials. It is considered as the prime cause for loosening of implant and also in total joint replacements where stress shielding of cortical bone is produced by a decade order of stiffer implant. The density of the commonly used implants and their alloys are also 2.5 to 4 times higher than the human bone, especially the cortical bone of which density varies from 1.8 to $2 \cdot 1 \mathrm{~g} / \mathrm{cc}$. These high-density metallic implants impart extra weight to the replaced part. Furthermore metals also corrode to some degree in the in vivo body environment.

Therefore, the main objective of the present work was to develop a polymer-ceramic composite and to characterize its mechanical properties. In this approach alumina powder will be reinforced in ultra high molecular weight polyethylene (UHMWPE) matrix. Alumina is a bio-inert material and widely used as load bearing articulating component design for its excellent wear and corrosion resistance (Chandra et al 1997), excellent biocompatibility (Kopecek and Ulibrich 1983) and high compressive strength. UHMWPE is a self-lubricating, lightweight, machinable and shaped by compression moulding, and it absorbs shock easily. The ceramic part will provide the strength (like mineral part of bone) and polymer, the resilience and flexibility similar to the collagenous part of the bone.

This research work may find application as a biomaterial in the health care industry. This type of composite will be suitable for non-weight bearing joints replacement, bone reconstruction, vertebral bodies, temporo-mandibular joints and in plastic surgery. So, the importance of this work is to replace the high cost foreign implants with indigenous implants by developing indigenous technology. 


\section{Experimental}

\subsection{Materials}

The ultra high molecular weight polyethylene used in the present work was Pilene ultra 2504 manufactured by National Organic Chemical India Ltd. (NOCIL). The molecular weight was in the range of 3.6 to 5.6 million as per the manufacturer data. The apparent density was measured to be 0.94 to $0.96 \mathrm{~g} / \mathrm{cc}$ for the powder samples and as well as for compressed cylindrical samples of length $15 \mathrm{~mm}$ and diameter $10 \mathrm{~mm}$. The alumina powder used for reinforcement was silane coated hollow sphere alumina oxide, SL-150, bubble cup, PQ Corporation, USA. The bulk density and the effective density are 0.4 and $0.77 \mathrm{~g} / \mathrm{cc}$, respectively as per literature of the supplier. The mean diameters of the powder particles were around $80 \mu \mathrm{m}$ and the SEM of the powder is shown in figure 1.

\subsection{Haemocompatibility}

The most important requirement for toxicity screening of biomaterial is haemocompatibility testing. The haemolysis test of the material was done as per ASTM protocol (F756-93). Test material was immersed in anticoagulated blood and incubated for $1 \mathrm{~h}$ at $37^{\circ} \mathrm{C}$, along with negative and positive controls. It was then centrifuged for $5 \mathrm{~min}$ at $500 \mathrm{~g}$ and the absorbance of the supernatant was determined at $545 \mathrm{~nm}$, using UV-spectrophotometer. The percentage haemolysis of UHMWPE and silane coated hollow sphere alumina powder were 3.92 and 4.89 , respectively. So both samples were haemo-compatible, as per ASTM.

\subsection{Methods}

In compression moulding, samples were prepared from a three-piece cylindrical mould designed by us. The internal

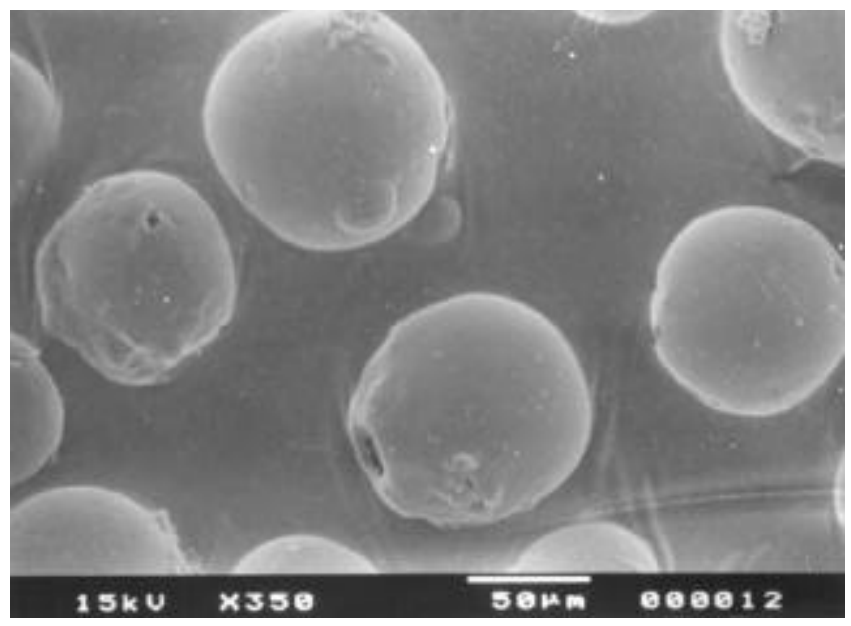

Figure 1. SEM of silane coated hollow sphere of alumina particles. and external diameters of the die were $10 \mathrm{~mm}$ and $20 \mathrm{~mm}$ respectively and length, $30 \mathrm{~mm}$. The outer diameters of the punch and bottom support are $9.5 \mathrm{~mm}$ and provide a gap of $0.5 \mathrm{~mm}$ between punch and die cavity, which is essential for such compression moulding. The samples were prepared by powder processing technique as suggested by the supplier, which involves cold compaction followed by sintering at polymer melting temperature.

The coated ceramic powders were uniformly mixed with UHMWPE in different weight percentages $(15 \%$, $20 \%, 25 \%, 30 \%, 35 \%, 40 \%, 45 \%$ and $50 \%$ ) of alumina oxide using a mixer grinder. The uniformly mixed powder is filled in the die cavity and is then kept inside a temperature-programmed heater. This total assembly was placed between the two compression plates of the universaltesting machine (Instron 4204). The powder was cold compressed for 5-10 min under a pressure of $8 \mathrm{MPa}$ to expel the air. This helps in heat conduction and leaves no void space after final compression. The temperature was raised to $180^{\circ} \mathrm{C}$ to melt the UHMWPE powder and held constant for $1 \mathrm{~h}$. During heating, the pressure gradually reduced and maintained at $2 \mathrm{MPa}$ at the melting stage. After heating for $1 \mathrm{~h}$, the pressure was increased and brought up to $8 \mathrm{MPa}$. This pressure was maintained until the total mass came back to the ambient temperature. About 60 samples were prepared for testing.

\section{Mechanical testing}

\subsection{Compression testing}

The moulded samples were squared up to the axis using low speed circular diamond-sawing machine (Make DUCOM). The opposite faces of the samples were made parallel. All specimens were immersed in normal saline and kept for 5-6 h. The specimens were cleaned and dried at $37^{\circ} \mathrm{C}$ in an incubator. Saline solution was isotonic to body fluid, which simulated in vivo fluid environment. This ensured similar moisture absorption for all samples. Compression test of the samples was conducted in the universal-testing machine Instron 4204 at a crosshead speed of $1 \mathrm{~mm} / \mathrm{min}$. The load vs deformation curve was recorded in the XY recorder from which the stress strain curve was prepared.

The nearly uniform distribution of particles in the composite was checked using reflection microscope and SEM is shown in figure 2. It also reveals how the alumina particles were embedded in the polymer matrix.

\subsection{Non-destructive testing}

The mechanical properties of the samples were also determined using ultrasound flaw detector machine (Matec SR-9000). Nearly $3 \mathrm{~mm}$ thick specimens were prepared from the sample and tested using $10 \mathrm{MHz}$ ultrasound 
transducer. The velocity of ultrasound through the specimen was calculated from the transmission time. The modulus of elasticity was determined from the ultrasonic transmission velocity and the density of the sample.

\section{Results}

From the stress vs strain diagram, we calculated the compressive yield strength and modulus of elasticity (static mechanical test). These values along with the corresponding density and modulus of elasticity from ultrasound testing (dynamic mechanical test) were shown in table 1. The mean yield strengths and mean modulii of elasticity in compression of the polyethylene and its composites are shown in figures 3 and 4 .

The legends for figures 3 and 4 indicate the samples as: Gr. $1=$ UHMWPE, Gr. $2=15 \mathrm{wt} \%$, Gr. $3=20 \mathrm{wt} \%$, Gr. $4=25 \mathrm{wt} \%$, Gr. 5=30wt $\%$, Gr. $6=35 \mathrm{wt} \%$, Gr.

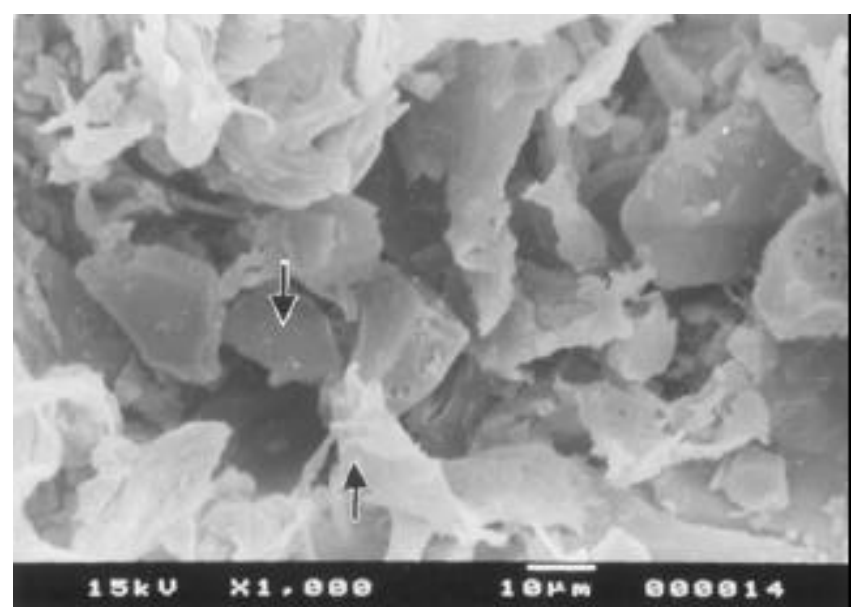

Figure 2. SEM of $40 \mathrm{wt} \%$ alumina reinforced composite ( $\downarrow$ for alumina and $\uparrow$ for polymer).
$7=40 \mathrm{wt} \%$, Gr. $8=45 \mathrm{wt} \%$ and Gr. $9=50 \mathrm{wt} \%$ of the filler alumina powder.

\section{Discussion}

From the above results it is revealed that the compressive strengths are dependent on the density of the composites. The compressive yield strength and modulus of elasticity increases progressively up to $45 \mathrm{wt} \%$ of alumina and then decreased. The modulus of elasticity and yield strength of the composite increased by $68 \%$ and $36 \%$, respectively as compared to the UHMWPE samples.

The modulus of elasticity obtained using ultrasound indicated nearly 8 times the values obtained from mechanical test. It is not unexpected, as the material is viscoelastic in nature and due to high rate of straining the material became stiffer. This value also increased linearly as with the density of the composite up to $45 \mathrm{wt} \%$ of reinforcement.

Recently, we tested some normal human cortical bone obtained from radius and cancellous bone from femoral head, collected from the orthopaedic department of a local hospital in Kolkata, during reconstructive surgery. The sizes were $10 \mathrm{~mm}$ cubes for cancellous bone and $6 \times 6 \times 10 \mathrm{~mm}^{3}$ for cortical bone. The mean ultimate compressive strength of cortical bone was $70 \mathrm{MPa}$ and that of cancellous bone $15 \mathrm{MPa}$ in the longitudinal direction. The corresponding modulii of elasticity for cortical and cancellous bones were $1.2 \mathrm{GPa}$ and $0.3 \mathrm{GPa}$, respectively (Ghosh 2001). These values were about $40 \%$ of standard western values. However, larger sample size is probably necessary for more reliable data.

The alumina particles penetrate into the polymer matrix and provide a greater stress transfer regime in the final composites. This in turn enhances the mechanical properties needed for strength retention by the non-resorbable polymer matrices. Low bulk density silane coated hollow

Table 1. Physical properties of UHMWPE and its composites with silane coated alumina*.

\begin{tabular}{|c|c|c|c|c|}
\hline & $\begin{array}{l}\text { Density } \\
(\mathrm{g} / \mathrm{cc})\end{array}$ & $\begin{array}{l}\text { Yield strength } \\
(\mathrm{MPa})\end{array}$ & $\begin{array}{l}\text { Modulus of elasticity } \\
\text { (Static mech. test) } \\
\text { (GPa) }\end{array}$ & $\begin{array}{c}\text { Modulus of elasticity } \\
\text { (Dynamic mech. test) } \\
\text { (GPa) }\end{array}$ \\
\hline Specimens & mean \pm s.d. & mean \pm s.d. & mean \pm s.d. & mean \pm s.d. \\
\hline UHMWPE & $0.94 \pm 0.02$ & $8 \cdot 26 \pm 0 \cdot 24$ & $0 \cdot 44 \pm 0 \cdot 09$ & $3.55 \pm 0.08$ \\
\hline $15 \% \mathrm{Al}_{2} \mathrm{O}_{3}$ & $0.96 \pm 0.01$ & $8 \cdot 43 \pm 0 \cdot 22$ & $0.51 \pm 0 \cdot 04$ & $4 \cdot 22 \pm 0 \cdot 11$ \\
\hline $20 \% \mathrm{Al}_{2} \mathrm{O}_{3}$ & $0.97 \pm 0.01$ & $8 \cdot 56 \pm 0 \cdot 37$ & $0.55 \pm 0 \cdot 04$ & $4 \cdot 47 \pm 0 \cdot 18$ \\
\hline $25 \% \mathrm{Al}_{2} \mathrm{O}_{3}$ & $1.01 \pm 0.04$ & $9 \cdot 12 \pm 0 \cdot 35$ & $0 \cdot 58 \pm 0 \cdot 04$ & $4 \cdot 62 \pm 0 \cdot 12$ \\
\hline $30 \% \mathrm{Al}_{2} \mathrm{O}_{3}$ & $1.04 \pm 0.01$ & $9 \cdot 43 \pm 0 \cdot 14$ & $0.59 \pm 0 \cdot 05$ & $4 \cdot 92 \pm 0 \cdot 19$ \\
\hline $35 \% \mathrm{Al}_{2} \mathrm{O}_{3}$ & $1.09 \pm 0.01$ & $9 \cdot 84 \pm 0 \cdot 12$ & $0.68 \pm 0.09$ & $5 \cdot 21 \pm 0 \cdot 14$ \\
\hline $40 \% \mathrm{Al}_{2} \mathrm{O}_{3}$ & $1 \cdot 12 \pm 0.02$ & $11 \cdot 03 \pm 0 \cdot 21$ & $0 \cdot 70 \pm 0 \cdot 05$ & $5 \cdot 37 \pm 0 \cdot 20$ \\
\hline $45 \% \mathrm{Al}_{2} \mathrm{O}_{3}$ & $1 \cdot 15 \pm 0.05$ & $11 \cdot 24 \pm 0 \cdot 31$ & $0.73 \pm 0.04$ & $5 \cdot 52 \pm 0 \cdot 21$ \\
\hline $50 \% \mathrm{Al}_{2} \mathrm{O}_{3}$ & $1 \cdot 21 \pm 0.04$ & $11 \cdot 01 \pm 0 \cdot 21$ & $0.72 \pm 0 \cdot 06$ & $5 \cdot 51 \pm 0 \cdot 19$ \\
\hline
\end{tabular}

*Six samples in each group, $p \leq 0 \cdot 006$. 


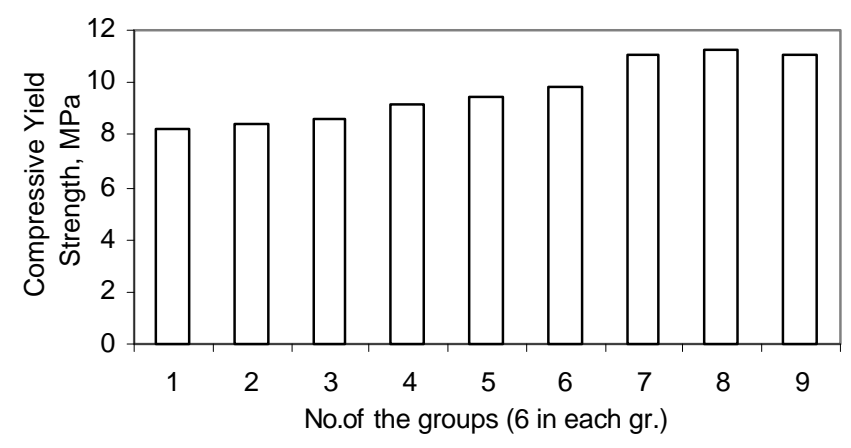

Figure 3. Mean yield strength comparison of UHMWPE with its various alumina wt\% composites (each group consists of 6 samples).

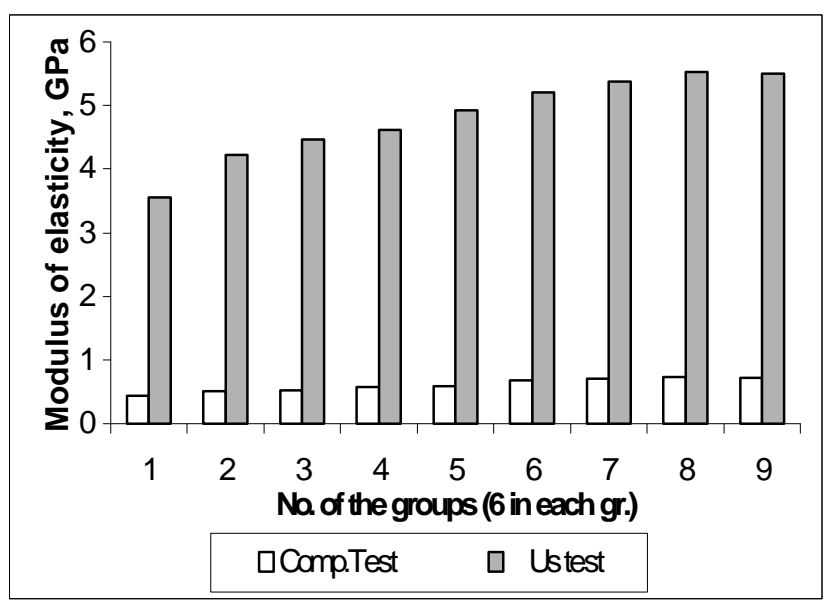

Figure 4. Mean modulus of elasticity comparison of UHMWPE with its various alumina wt $\%$ composites (each group consists of 6 samples).

sphere alumina did not settle down during composite processing and remain nearly uniformly distributed in the final product, unlike normal alumina powder.

The density of the composite increased by $22 \%$ for $45 \mathrm{wt} \%$ alumina in comparison to ultra high molecular weight polyethylene. This value is close to the human bone. All samples were compressed in the Universal testing machine. A crack was formed only in $50 \mathrm{wt} \%$ sample at high stress, but other samples did not crack.

\section{Conclusions}

The load carrying capacity of UHMWPE was improved by addition of silane coated hollow sphere alumina ceramic. The reinforcing alumina supports a significant amount of load and this is a distinct advantage of using this composite in medicine. The derived properties of these composites are close to the human bone, which we tested earlier. These improved physical properties will not hopefully cause any strain incompatibility, when it will be used as a substitute for metal or its alloy prostheses. Though, the physical properties of the composites are closer to natural bone, it is essential to study the biocompatibility before use as a fracture fixation plate or as a minor load bearing joint. Further evaluations are continuing to determine its dynamic properties and long-term sustainability in vivo environment.

\section{Acknowledgements}

A part of this work was supported by the AICTE grant to the School of Bioscience and Engineering 1997-2000. Dr C Basu, IACS, Kolkata, is also acknowledged for her assistance.

\section{References}

Annual Book of ASTM Standard 1996 Medical Devices: Sec 13, Vol. 13.01

Bonfield W 1988 Bioceramics $\mathbf{5 2 3} 173$

Chandra A, Mukhopadhyay K A, Basu D and Chatterjee S 1997 Ceram. Int. 23437

Ghosh D 2001 Studies on fluoride contamination of human bone and its characteristics, Masters Thesis, Jadavpur University, Kolkata

Kopecek J and Ulibrich K 1983 Biodegradation of biomedical polymers; Progress in polymer science (New York: Pergamon) 9 pp 2-58

Park J B 1984 Biomaterials science and engineering (New York: Plenum Press) p. 135

Ravglioli A and Krajewski A 1992 Bioceramics (London: Chapman and Hall) p. 44

Woo S L Y et al 1976 J. Bone Jt. Surg. A58 190 